WTERFFAET TECNOLÓGGCA

\title{
LOGÍSTICA REVERSA E SUSTENTABILIDADE NA REUTILIZAÇÃO DE EMBALAGEM BIG BAG
}

\section{REVERSE LOGISTICS AND SUSTAINABILITY IN THE REUSE OF BIG BAG PACKAGING}

\author{
Ivan dos Santos - ivanmvp9@gmail.com \\ Paulo Henrique Benedeti - paulohbenedeti@gmail.com \\ Rhadler Herculani - rhadler.herculani@fatec.sp.gov.b \\ Faculdade de Tecnologia de Bebedouro (FATEC) - SP - Brasil
}

DOI: 10.31510/infa.v15i2.512

\begin{abstract}
RESUMO
O presente artigo visa comprovar a geração de valor na cadeia reversa de suprimentos da recuperação e higienização de contentores flexíveis (Big Bags), que são embalagens utilizadas no acondicionamento, armazenamento e transporte de produtos. A logística reversa, embora sempre associada a reciclagem de materiais, possui um conceito mais amplo, pois se associa também se relaciona com atividades logísticas de redução de fontes de abastecimento, substituição, reuso de materiais e disposição. Este processo propicia benefícios financeiros na redução de custos, impacto positivo ao meio ambiente e na imagem organizacional perante seus fornecedores, clientes e, consequentemente, para a sociedade. Utilizou-se os métodos de pesquisa bibliográfica, estudo de caso e análise de caráter qualitativo para o desenvolvimento do artigo. Os resultados obtidos se mostraram satisfatórios de acordo com as informações analisadas conforme objetivo proposto. Conclui-se que a logística reversa afeta diretamente os resultados da organização proporciona grande satisfação dos clientes, contribui para o desenvolvimento sustentável e estimula uma cooperação mútua entre os parceiros envolvidos na cadeia reversa.
\end{abstract}

Palavras chave: Cadeia Reversa de Suprimentos. Geração de Valor. Contentor flexível.

\begin{abstract}
The objective of this article is to show the creation of value in the reverse supply chain in recovery and cleanness of flexible containers (Big Bags), which are used in the packaging, storage and transport of products. Reverse logistics, although always associated with material recycling, has a broader concept, since it is also associated with logistics activities related to reduction of sources of supply, substitution, reuse of materials and disposal.This process provides financial benefits in the reduction of costs, positive impact on the environment and on the organizational image before its suppliers, customers and, consequently, to society. We used the methods of bibliographic research, case study and qualitative analysis for the development
\end{abstract}




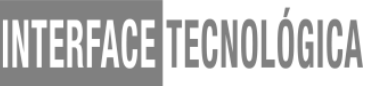

of the article. The results obtained were satisfactory according to the information analyzed according to the proposed objective. It is concluded that reverse logistics directly affect the organization's results, provides great customer satisfaction, contributes to sustainable development and encourages mutual cooperation between the partners involved in the reverse chain.

Keywords: Reverse Supply Chain. Value Generation. Flexible Container.

\section{INTRODUÇÃO}

A globalização, a exigência dos clientes, legislação dos governos, e outros fatores fazem com que as organizações tenham que estar sempre em desenvolvimento para manter seu espaço e competitividade. A utilização da logística reversa pode trazer grandes resultados; de acordo com Campos (2003), um eficiente sistema da cadeia reversa de suprimentos pode transformar algo de grande custo em vantagem competitiva.

Conforme Leite (2003), o aumento do interesse pela logística reversa vem da crescente preocupação com o meio ambiente e principalmente em atender os anseios dos clientes, fornecedores, visando sempre a minimização de custos. Buscar a sustentabilidade corporativa requer foco no desenvolvimento e aprimoramento de três macro objetivos, que Pedroso e Zwicker (2007), definem como: i) geração de valor econômico; ii) responsabilidade ambiental; e a iii) responsabilidade social. Leite (2003) indica a logística reversa como a oportunidade de gerar valor para cadeia de suprimentos, dando nova destinação a bens utilizados, mas que ainda podem ser reaproveitados, ou seja possuem valor.

Utilizando-se da metodologia de estudo de caso, baseando-se no levantamento de dados coletados com os agentes da cadeia reversa de suprimentos, o trabalho busca resposta para o seguinte problema: é possível o sistema reverso de embalagens big bag ser sustentável?

Portanto, o objetivo do presente artigo é comprovar a geração de valor na cadeia reversa de suprimentos da recuperação e higienização de contentores flexíveis (Big Bags), que são embalagens utilizadas no acondicionamento, armazenamento e transporte de produtos. Este estudo se faz necessário para evidenciar que a aplicação da Logística Reversa eficiente pode tornar uma empresa sustentável e, desta maneira, difundir conhecimento sobre os tópicos relacionados para a conscientização organizacional. 


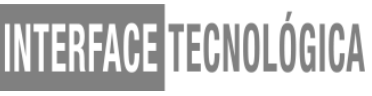

A relevância do artigo também procura fomentar o estímulo a novos estudos na área em questão para que cada vez mais o ambiente organizacional se torne sustentável.

\section{FUNDAMENTAÇÃO TEÓRICA}

\subsection{Logística reversa}

Geralmente, quando se fala em logística reversa, associa-se a reciclagem de materiais. Isso pode ter origem com a primeira publicação de definição do termo efetuado pelo Council of Logistics Management-CLM (Conselho de Gerenciamento Logístico, no início dos anos 1990:

[...] logística reversa é o termo geralmente usado para referir o papel da logística na reciclagem, disposição de resíduos e gerenciamento de materiais perigosos; uma perspectiva mais ampla se relaciona com atividades logísticas de redução de fontes de abastecimento, reciclagem, substituição, reuso de materiais e disposição.

Leite (2003) define a logística reversa como a área responsável pelos fluxos provenientes do retorno dos bens de pós-venda e pós consumo aos ciclos de negócios, dar uma nova destinação via mercado secundário, ou via ciclos produtivos devolvendo a produção da própria organização, através da distribuição reversa. Complementando, Adlmaier e Sellito (2007), dizem que essa reintegração se dá sob a forma de insumo ou matéria-prima e definem três etapas para as atividades de logística reversa: i)processamento do fluxo reverso das mercadorias por danos, produtos vencidos ou fora de época em determinado local necessitando ser realocado; ii) reciclagem ou reaproveitamento das embalagens, recondicionamento direto dos produtos ou necessita de remanufatura, definir o status para saber onde encaminhá-lo; e, iii) descartes de equipamentos velhos sem utilidade, controle de materiais perigosos ou recuperação de patrimônio. Dentro dessas atividades do ciclo de distribuição reverso pode-se agregar diversos valores aos produtos e materiais. Para Leite (2003) podem ser valores econômicos, ecológicos, legais, logísticos, e até de imagem corporativa.

Um sistema de logística reversa deve considerar alguns fatores críticos que são: a) planejamento e aplicação de bons controles de entrada, que direciona corretamente os materiais para cada fluxo (reutilização, reciclagem, recondicionamento e revenda); b) padronização; c) 


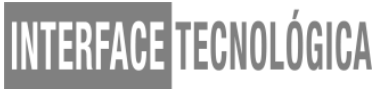

mapeamento de processos; d) qualidade do serviço; e) rápida identificação de necessidades. Tais fatores tornam o processo mais eficiente e reduzem custos (LACERDA, 2009).

\subsection{Cadeia Reversa de Suprimentos}

Em busca de uma eficiência e melhoria continua na geração de valor empresarial surge a Gestão da Cadeia de Suprimentos (Supply Chain Management - SCM), definida, como um conjunto de abordagens que integra, com eficiência e eficácia, fornecedores, fabricantes, depósitos e pontos comerciais, atendendo as exigências nos níveis de serviço e na diminuição de custos, através da produção e distribuição na quantidade certa, entrega dentro do prazo e com a qualidade demandada (SIMCHI-LEVI et al., 2010). As cadeias de suprimentos têm como objetivo a maximização da geração de valor global (CHOPRA; MEINDL, 2003).

A fase de elaboração de um canal reverso é definida pelas práticas e procedimentos organizacionais envolvidos em todas as etapas de retorno dos materiais ou produtos, pela troca de informações e um bom relacionamento entre as empresas incluídas na cadeia e pelo grau de recursos inseridos nas operações desenvolvidas e executadas (LEITE, 2003). Compreendendo o sistema da cadeia reversa, De Brito e Dekker (2002) descrevem três perguntas essenciais para analisar a estrutura das cadeias reversas: i) “por quê?”, motivos e forças que apontam o retorno; ii) "o quê?": características do produto para retorno; e iii) “como?”: estruturação do processo de recuperação.

\subsection{Sustentabilidade}

A humanidade está passando por fases de inconstância sistêmica, gerada pela preocupação entre a globalização econômica, cultural, e a emergência dos limites ambientais, reivindicando a transição para uma sociedade sustentável (MANZINI; VEZZOLI, 2002).

Para Savitz e Webber (2007), sustentabilidade deve envolver no planejamento e tomada de decisões não apenas aspectos econômicos, mas considerar também as responsabilidades sociais e ambientais, e manter um equilíbrio em relação entre estes três pilares. Conhecidos como Triple Bottom Line (TBL), criado por Elkington (1994), onde afirma que a empresa sustentável é a que consegue proporcionar valores e benefícios nestes três pilares. Nesse contexto Pedroso e Zwicker (2007), consideram que valor econômico está relacionado em obter lucros no presente e no futuro, responsabilidade social abrange contribuir com o 


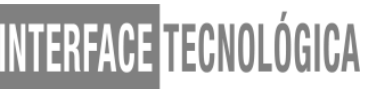

desenvolvimento da sociedade e responsabilidade ambiental é a conservação, manutenção dos recursos naturais utilizando-os de forma sustentável. Conclui-se que para alcançar a sustentabilidade corporativa necessita obter equilíbrio econômico, social e ambiental. A figura 1 sugere uma estrutura para perspectiva de casos de sustentabilidade corporativa.

Figura 1: Sustentabilidade Corporativa

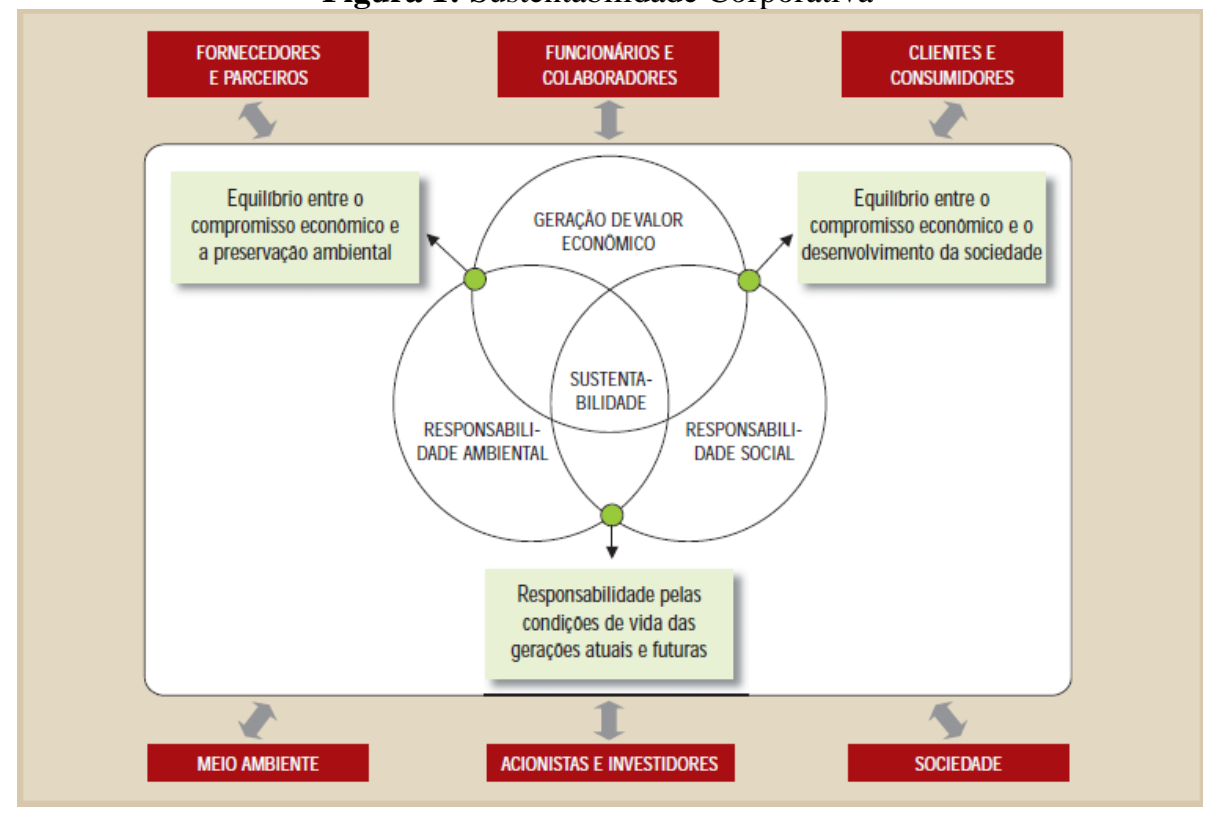

Fonte: Pedroso (2007)

\subsection{Embalagens retornáveis (reutilizáveis)}

Neste cenário de alta competitividade encontrar formas de conseguir obter uma vantagem competitiva pode ser encontrada em algo indispensável para o produto, a embalagem. Embalagens retornáveis podem gerar economia e com isso prover ganhos, incitar iniciativas e esforços no desenvolvimento e melhoria dos processos de logística reversa (CHAVES; BATALHA, 2006). Um processo reverso eficiente de embalagens pode propiciar resultados significativos podendo gerar novas oportunidades de práticas sustentáveis.

As embalagens ao passarem pelas funções de transportes, contenções e proteções podem retornar ao processo produtivo, sem ter que sofrer um processo de transformação do seu estado físico (NOVAES, 2009). Contudo, é importante salientar que dependendo do tipo de embalagem e em qual tipo de indústria e processo ela seja utilizada, será necessário passar por um processo de higienização antes de sua reutilização, atendendo as normas de segurança alimentar e qualidade. As empresas que efetuam esse trabalho de higienização precisam atender 
em seus processos as normas de BPF (Boas Práticas de Fabricação para a indústria alimentícia), já as empresas que são fabricantes de embalagens reutilizáveis devem atender integralmente as normas da ABNT (Associação Brasileira de Normas Técnicas), e atender às exigências definidas nos regulamentos técnicos em vigor da ANVISA (Agência Nacional de Vigilância Sanitária) e estarem devidamente licenciadas junto ao órgão de vigilância sanitária de sua região.

\subsubsection{Contentor Flexível Big Bag}

Big bag é uma embalagem flexível super-resistente, versátil, econômica, possibilita facilidade com a armazenagem e transporte de produtos na forma sólida. Esta embalagem possui dois modelos para comercialização o tipo One Way que são descartáveis, e, o tipo Multi Way que que são reutilizáveis. O quadro abaixo demonstra algumas vantagens da escolha por utilização de big bag.

\begin{tabular}{|l|}
\multicolumn{1}{c|}{ Quadro 1 - Vantagens Big Bag } \\
\hline \multicolumn{1}{|c|}{ Vantagens big bag } \\
\hline$\checkmark$ Embalagens extremamente econômicas \\
\hline$\checkmark$ Fáceis de manusear, otimizam as operações logísticas dos seus clientes \\
\hline$\checkmark$ Cumprem inteiramente as normas de higiene estritas a nível da indústria alimentar \\
\hline$\checkmark$ Podem transportar até mil vezes o seu próprio peso em segurança \\
\hline$\checkmark$ Não necessitam de uma segunda embalagem \\
\hline$\checkmark$ Peso reduzido, menor espaço de ocupação - economia no armazenamento \\
\hline$\checkmark$ Ajudam a minimizar os fluxos de resíduos \\
\hline$\checkmark$ Reciclagem e recondicionamento \\
\hline
\end{tabular}

Fonte: adaptado de LC Packaging (2018)

\section{PROCEDIMENTOS METODOLÓGICOS}

O presente trabalho é fundamentando por meio de pesquisa exploratória, que segundo Gil (2002), busca proporcionar maior familiaridade com o problema, buscando torna-lo mais explícito. A metodologia utilizada é a de estudo de caso, que segundo Yin, (2005), é adequado quando se pretende investigar o como e por quê de um conjunto de eventos contemporâneos. Bruyne, Herman e Schoutheete (1977), afirmam que o estudo de caso justifica sua importância por juntar informações detalhadas que contribuem para o aprendizado total de uma situação. 


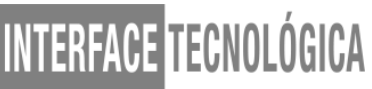

O estudo de caso deste artigo contempla informações sobre os processos, métodos utilizados no retorno de embalagens big bag para o processo produtivo das usinas de açúcar do interior de São Paulo, especificamente na área de São José do Rio Preto e Ribeirão Preto, também faz parte do estudo informações dos prestadores de serviços que participam dos processos dos fluxos reversos, e por questões de segurança e preservação da imagem não permitiram as divulgações das imagens. As informações foram obtidas através de relatórios e diálogos com profissionais que responsáveis pela gestão de logística reversa, bem como profissionais que prestam serviços dentro do ciclo do canal reverso. Estas informações agrupadas geraram os quadros que compõem o banco de dados do artigo.

\section{RESULTADOS E DISCUSSÃO}

A produção de açúcar no Brasil na safra de 2017/2018 foi de 37,87 milhões de toneladas, de acordo com dados e relatórios publicados pelo Ministério da Agricultura (2018), deste valor $74,6 \%$ foi produzido na região sudeste do país, tendo como estado com maior produção o de São Paulo que foi responsável por $84,75 \%$ do total produzido na região, isto representa aproximadamente 23,85 milhões de toneladas de açúcar. A armazenagem, transporte e comércio desse produto para os mercados industriais são utilizados na maior parte da produção a embalagem big bag multi way, que de acordo com profissionais de logística do setor propicia muitos benefícios e facilidades para toda a cadeia, por ser uma embalagem com característica de unitizador dispensa uma embalagem secundária, seu manuseio pode ser feito por meio de empilhadeiras, pontes rolantes ou talha, além de mecanismos automatizados como o envaze, seu material flexível e altamente resistente possibilita a estocagem em forma de pirâmide, onde o big bag é colocado sobre outros dispensando estruturas para estoques, também torna o carregamento e descarregamento de caminhões mais ágil e o transporte mais seguro. Todos esses fatores contribuem para a eficiência dentro da cadeia produtiva e de distribuição.

Após o envio para os clientes entra em cena a logística reversa que terá a responsabilidade de realizar os controles e acompanhamento das devoluções dos clientes e posteriormente o envio para as lavanderias de higienização, para que esse fluxo ocorra de forma eficiente e eficaz algumas medidas são necessárias. Dentre elas: um fluxo de informações confiáveis e contínuas entre os participantes do fluxo reverso; flexibilidade e agilidade para resolver problemas não previstos no planejamento; e conhecimento amplo sobre as legislações 
em vigor. A logística reversa busca extrair o máximo de valor da embalagem, realizando sua parte na sustentabilidade corporativa.

Figura 2: Fluxograma cadeia direta e reversa do big bag

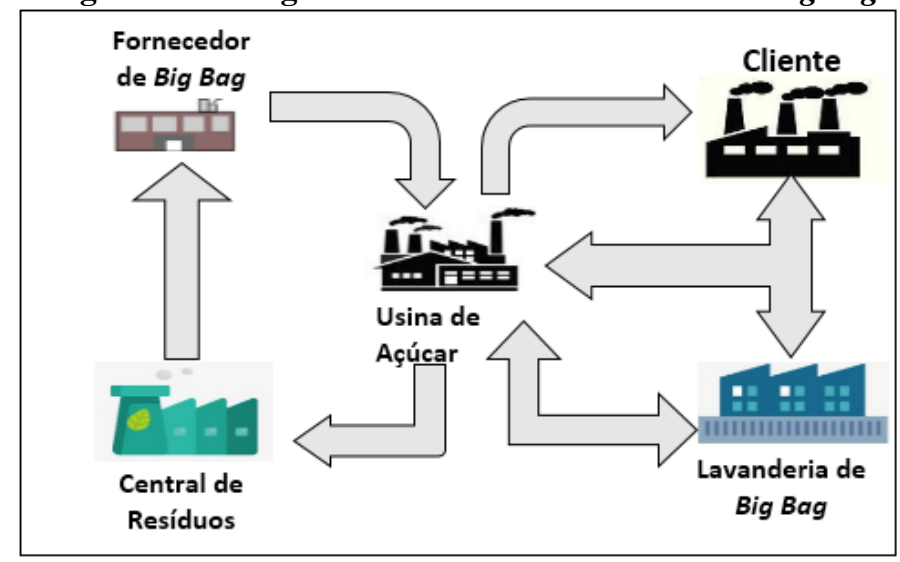

Fonte: elaborado pelo autor

A passagem do big bag pelos agentes integrantes da cadeia reversa é representando na figura 2 que ilustra o caminho percorrido pela embalagem big bag desde a compra até o seu retorno para o fornecedor.

Para entender melhor a utilização, benefícios e vantagens propiciados ao adotar a utilização deste tipo de embalagem, o quadro 2 contém dados levantados por meio de relatórios com alguns profissionais que atuam nesse setor em usinas produtoras de açúcar.

O quadro 2 contempla informações e dados de nove usinas.

Quadro 2 - Dados e Informações Usinas de Açúcar

\begin{tabular}{|c|c|c|c|}
\hline \multicolumn{4}{|c|}{ Usinas de Açúcar } \\
\hline Tipo de Big Bag & Tópico & Quantificação & Observação \\
\hline \multirow{6}{*}{ Multi way } & Preço Médio & $\mathrm{R} \$ \quad 52,00$ & \multirow{6}{*}{$\begin{array}{c}\text { Utilizado para } \\
\text { mercado industrial } \\
\text { interno, estoque e } \\
\text { armazenagem para } \\
\text { reprocessamento } \\
\text { para produção de } \\
\text { fardos }\end{array}$} \\
\hline & Giro Médio por Safra & 2 vezes & \\
\hline & Vida Útil & 5 anos & \\
\hline & Volume/Quantidade Médio & 780.000 & \\
\hline & Volume de Produção Envasada & $70 \%$ & \\
\hline & Percentual de Descarte por Safra & $7 \%$ & \\
\hline \multirow{5}{*}{ One way } & Preço Médio & $\mathrm{R} \$ \quad 35,00$ & \multirow{5}{*}{$\begin{array}{l}\text { Utilizados para } \\
\text { exportação, } \\
\text { produção somente } \\
\text { sobre demanda }\end{array}$} \\
\hline & Giro Médio por Safra & $1 \mathrm{vez}$ & \\
\hline & Vida Útil & Descartável & \\
\hline & Volume/Quantidade Médio & - & \\
\hline & Volume de Produção Envasada & - & \\
\hline
\end{tabular}

Fonte: elaborado pelo autor

Através desse quadro pode-se verificar o grande volume de big bags necessários para atender a demanda das usinas, bem como seu preço médio. 
|WTEREFAEETECNOLOGGCA

Quadro 3 - Dados e Informações Lavanderias Big Bag

\begin{tabular}{|c|c|c|c|}
\hline \multicolumn{4}{|c|}{ Lavanderia } \\
\hline Etapa da Produção & Tópico & Quantificação & Observação \\
\hline \multirow{3}{*}{ Produção Total } & Preço Médio & $\mathrm{R} \$ \quad 3,50$ & \multirow{11}{*}{$\begin{array}{c}\text { Todo big bag é } \\
\text { higienizado antes de } \\
\text { ser inspecionados e } \\
\text { serem classificados } \\
\text { como: Pronto, } \\
\text { Manutenção, } \\
\text { Descarte. } \\
\text { (Descarte retorna } \\
\text { para o cliente } \\
\text { também para } \\
\text { posteriormente ser } \\
\text { encaminhado para } \\
\text { central de resíduos) }\end{array}$} \\
\hline & Volume Processado & 740.000 & \\
\hline & Percentual da Produção & $100 \%$ & \\
\hline \multirow{2}{*}{ Higienização } & Volume Processado & 740.000 & \\
\hline & Percentual da Produção & $100 \%$ & \\
\hline \multirow{2}{*}{ Manutenção } & Volume Processado & 297.600 & \\
\hline & Percentual da Produção & $40 \%$ & \\
\hline \multirow{2}{*}{ Descarte } & Volume Processado & 51.750 & \\
\hline & Percentual da Produção & $7 \%$ & \\
\hline \multirow{2}{*}{ Funcionários } & Diretos & 120 & \\
\hline & Indiretos & 60 & \\
\hline
\end{tabular}

Fonte: elaborado pelo autor

A coleta de dados também ocorreu nas lavanderias que prestam serviços para as usinas, o quadro 3 contempla dados de duas lavanderias que realizam o serviço de higienização das embalagens, atendendo as normas de qualidade e segurança do alimento.

Conforme o quadro 4, o cálculo de uma simulação para mostrar em valores a economia gerada pelas embalagens reutilizáveis.

Quadro 4 - Avaliação utilização big bag one way X multi way

\begin{tabular}{|c|c|c|c|c|c|c|c|c|c|c|c|}
\hline \multicolumn{12}{|c|}{ Valores em $\mathrm{R} \$$} \\
\hline \multicolumn{2}{|c|}{ Número da Utilização } & 1 & 2 & 3 & 4 & 5 & 6 & 7 & 8 & 9 & 10 \\
\hline \multirow{5}{*}{$\begin{array}{l}\text { ONE } \\
W A Y\end{array}$} & Necessidade de compra & Sim & Sim & Sim & Sim & Sim & Sim & Sim & Sim & Sim & Sim \\
\hline & Preço de compra & -35 & -35 & -35 & -35 & -35 & -35 & -35 & -35 & -35 & -35 \\
\hline & Transporte de retorno & $-2,5$ & $-2,5$ & $-2,5$ & $-2,5$ & $-2,5$ & $-2,5$ & $-2,5$ & $-2,5$ & $-2,5$ & $-2,5$ \\
\hline & Higienização & $\begin{array}{ll}-- \\
-\end{array}$ & --- & --- & --- & --- & --- & --- & --- & --- & --- \\
\hline & Valor de venda descarte & 0,5 & 0,5 & 0,5 & 0,5 & 0,5 & 0,5 & 0,5 & 0,5 & 0,5 & 0,5 \\
\hline \multicolumn{2}{|c|}{ Total acumulado por utilização } & -37 & -74 & -111 & -148 & -185 & -222 & -259 & -296 & -333 & -370 \\
\hline \multirow{5}{*}{$\begin{array}{c}\text { MULTI } \\
W A Y\end{array}$} & Necessidade de compra & Sim & Não & Não & Não & Não & Não & Não & Não & Não & Não \\
\hline & Preço de compra & -52 & --- & --- & --- & --- & --- & --- & $-\cdots$ & --- & $-\cdots$ \\
\hline & Transporte de retorno & $-2,5$ & $-2,5$ & $-2,5$ & $-2,5$ & $-2,5$ & $-2,5$ & $-2,5$ & $-2,5$ & $-2,5$ & $-2,5$ \\
\hline & Higienização & $-3,5$ & $-3,5$ & $-3,5$ & $-3,5$ & $-3,5$ & $-3,5$ & $-3,5$ & $-3,5$ & $-3,5$ & $-3,5$ \\
\hline & Valor de venda descarte & --- & --- & --- & --- & --- & --- & -- & --- & -- & 0,5 \\
\hline \multicolumn{2}{|c|}{ Total acumulado por utilização } & -58 & -64 & -70 & -76 & -82 & -88 & -94 & -100 & -106 & -112 \\
\hline
\end{tabular}

Fonte: elaborado pelo autor

Para conseguir a realização da simulação foi necessário a coleta de informações do custo de retorno dos big bags junto as usinas anteriormente consultadas e, também, transportadoras que realizam o transporte, os valores são baseados na média das empresas pesquisadas.

Valor econômico: A economia gerada com a reutilização das embalagens proporciona uma maior competitividade para as usinas, diminuindo seus custos de forma considerável, conforme o quadro a 4 mostra após 10 utilizações um big bag one way iria gerar um custo de 


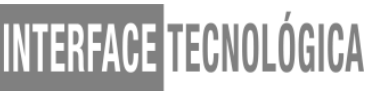

$\mathrm{R} \$ 370,00$ enquanto os reutilizáveis um custo de apenas $\mathrm{R} \$ 112,00$, uma economia no valor de $\mathrm{R} \$ 258,00$ por big bag.

Valor ambiental: A reutilização proporciona economia de matéria-prima, neste caso específico em média 10 vezes menos, e ainda após não ter mais utilidade, sendo considerado descarte o contentor é encaminhado para centrais de resíduos que o transforma em rezina para posteriormente voltar a cadeia produtiva do fabricante.

Valor social: A geração de valores sociais está presente nas lavanderias e centrais de resíduos por meio da geração de empregos diretos e indiretos, possibilitando assim uma maior circulação e distribuição de renda.

\section{CONCLUSÃO}

A logística reversa conforme mostrado nos tópicos anteriores tem uma importância crescente dentro das organizações, observa-se que isto é ocasionado pelo ponto de vista estratégico e econômico para alguns setores empresariais. Com o aumento da exigência e competitividade do mercado, esta área pode proporcionar grandes vantagens para as organizações, para isto é fundamental que os gestores e gerentes da cadeia de suprimentos tenham essa visão e destinem o mesmo foco e atenção que é dado a logística direta. Desta forma conclui-se que a logística reversa afeta diretamente os resultados da organização, pode propiciar grande satisfação dos clientes, contribui para o desenvolvimento sustentável, e por fim estimula uma cooperação mútua entre os parceiros envolvidos na cadeia reversa.

Quanto ao problema de pesquisa que impulsionou o interesse e desenvolvimento do trabalho, obteve-se um resultado positivo, que conforme constatado o sistema de reutilização de big bag é um sistema sustentável, porém há muitas possibilidades de melhorias que podem ser efetuadas, considerando que os ganhos trazidos pela economia de embalagens reutilizáveis cada vez mais incentivam novas iniciativas.

O artigo busca contribuir com o desenvolvimento do assunto e incentivar novas pesquisas sobre embalagens reutilizáveis big bag, pois não existem muitos trabalhos específicos a respeito desta embalagem que está crescendo no mercado brasileiro, como sugestão para a continuidade do trabalho, ser realizado um estudo mais específico englobando toda a cadeia, buscando mostrar os processos e práticas de cada um dos integrantes, verificando se estão alinhadas com a sustentabilidade e não apenas com a questão econômica. O caminho do sucesso é a estrada que leva a sustentabilidade, mesmo sendo o percurso mais difícil é o que traz o resultado vitorioso. 


\section{REFERÊNCIAS}

ADLMAIER, D.; SELLITTO, M. A. Embalagens retornáveis para transporte de bens manufaturados: um estudo de caso em logística reversa. Produção, v. 17, n.2. 2007.

BRUYNE, P.; HERMAN, J.; SCHOUTHEETE, M. Dinâmica da pesquisa em ciências sociais: os polos da prática metodológica. Rio de Janeiro: F. Alves, 1977. 251 p.

CAMPOS, T. Logística Reversa: aplicação ao problema das embalagens da CEAGESP. 2006. 154 f. Dissertação (MESTRADO) - Escola Politécnica, Universidade de São Paulo, São Paulo, 2006.

CHAVES, G. L. D., BATALHA, M. O. Os consumidores valorizam a coleta de embalagens recicláveis? Um estudo de caso da logística reversa em uma rede de hipermercados. Revista Gestão e Produção, vol.13, n.3, pp.423-434, 2006. Disponível em:

$<$ http://www.scielo.br/scielo.php?pid=S0104-

530X2006000300006\&script=sci_abstract\&tlng=pt>. Acesso em: 19 ago. 2018.

CHOPRA, S.; MEINDL, P. Gerenciamento da Cadeia de Suprimentos: estratégias, planejamento e operação. São Paulo: Person, 2003.

DE BRITO, M. P.; DEKKER, R. Reverse logistics: a framework. Econometric Institute. Report EI 2002-38, Erasmus University Rotterdam, The Netherlands, 2002.

DORNIER, P. et al. Logística e Operações Globais. São Paulo: Atlas, 2000.

ELKINGTON, J. Triple bottom line revolution: reporting for the third millennium. Australian CPA, v. 69, p. 75, 1994.

GIL, A. C. Como elaborar projetos de pesquisa. 4. ed. São Paulo: Atlas, 2002.

KOPICKI, R.; BERG, M.; LEGG, L. L. Reuse and Recycling: reverse logistics opportunities. Illinois: Oak Brook, Council of Logistics Management, 1993.

LACERDA, L. Logística reversa, uma visão sobre os conceitos básicos e as práticas operacionais. Centro de Estudos em Logística - COPPEAD - UFRJ - 2002. Disponível em:< http://www.paulorodrigues.pro.br/arquivos/Logistica_Reversa_LGC.pdf> Acesso em: 19 ago. 2018.

LC PACKAGING. As vantagens de um big bag. Disponível em: < https://bigbags.com/ptpt/porque-os-nossos-big-bags/vantagens-de-um-big-bag/> . Acesso em: 02 set. 2018.

LEITE, P.R. Logistica Reversa: meio ambiente e competividade. São Paulo: Prentice Hall, 2003. 
MANZINI, E.; VEZZOLI, C. O desenvolvimento de produtos sustentáveis. São Paulo: Editora da Universidade, 2002.

MINISTÉRIO DA AGRICULURA, PECUÀRIA E ABASTECIMENTO: Relatório Produção de Açúcar por Tipo - Safra 2017/18: Disponível em:

$<$ http://www.agricultura.gov.br/assuntos/sustentabilidade/agroenergia/arquivos-producao)>. Acesso em 08 set. 2018.

NOVAES, F. A logística reversa de caixas de papelão e seu impacto ambiental. Revista Gestão \& Saúde. Curitiba, 2009.

PEDROSO, M.C. Casos sustentáveis. GV-executivo, São Paulo, v.6, n.2, p.24-29, mar./abr. 2007.

PEDROSO, M.C; ZWICKER, R. Sustentabilidade na cadeia reversa de suprimentos: um estudo de caso do Projeto Plasma. Revista de Administração - RAUSP, vol. 42, núm. 4, octubre-diciembre, 2007, pp. 414-430. Universidade de São Paulo, São Paulo, 2007.

SAVITZ, A.W.; WEBER, K. A empresa sustentável: o verdadeiro sucesso é o lucro com responsabilidade social e ambiental. Rio de Janeiro: Elsevier, 2007.

SIMCHI-LEVI, D.; KAMINSKY, P.; SIMCHI-LEVI, E. Cadeia de Suprimentos Projetos e Gestão. $3^{\text {a }}$ ed. Porto Alegre: Bookman, 2010.

YIN, R. K. Estudo de caso: planejamento e métodos. 3. ed. Porto Alegre: Bookman, 2005. 